\title{
Gallium-67 Scan with Single Photon Emission Computed Tomography for the Evaluation and Monitoring of Infected Abdominal Aortic Aneurysms: A 10-Year Case Series
}

\author{
Hoi-Ming Kwok, Wing-Hang Luk, Lik-Fai Cheng, Nin-Yuan Pan, Ho-Fung Chan, \\ and Johnny Ka-Fai Ma \\ Department of Diagnostic and Interventional Radiology, Princess Margaret Hospital, Hong Kong, China
}

Purpose: This study aimed to evaluate the role of gallium-67 single photon emission computed tomography (SPECT) with contrast computed tomography (CT) in the evaluation and monitoring of infected abdominal aortic aneurysms (IAAA).

Materials and Methods: A retrospective cohort analysis was performed using prospectively collected data of consecutive patients with IAAA in Princess Margaret Hospital in Hong Kong between January 2010 and December 2020. The patients were identified using the Radiology Information System.

Results: All five patients had proven IAAA on CT and/or metabolic imaging. Among them, three were further supported by positive blood culture results. Gallium-67 SPECT with contrast CT was useful in the detection of residual disease, monitoring, and detection of recurrence.

Conclusion: Gallium-67 SPECT with contrast CT is helpful for evaluating IAAA. It serves an important role in guiding management, especially during long-term follow-up.

Key Words: Gallium isotope, Single photon emission computed tomography scan, Infected aneurysm, Abdominal aortic aneurysm, Diagnostic imaging
Received March 15, 2021

Revised May 19, 2021

Accepted June 14, 2021

Published on June 29, 2021

Corresponding author: Hoi Ming Kwok

Department of Diagnostic and Interventional Radiology, Princess

Margaret Hospital, 2-10 Princess Margaret Hospital Road, Lai Chi Kok, Hong Kong, China

Tel: 852-2990-1111

Fax: 852-2786-3629

Email: hmkwok15@hotmail.com https://orcid.org/0000-0001-7628-1687

Copyright (c) 2021 The Korean Society for Vascular Surgery

This is an Open Access article distributed under the terms of the Creative Commons Attribution Non-Commercial License (http://creativecommons.org/licenses/by-nc/4.0) which permits unrestricted non-commercial use, distribution, and reproduction in any medium, provided the original work is properly cited.

Vasc Specialist Int • https://doi.org/10.5758/vsi.210020

\section{INTRODUCTION}

An infected aneurysm is defined as an infectious break in the arterial wall with the formation of a blind, saccular outpouching that is contiguous with the arterial lumen. The prevalence is estimated to be around 0.5\% [1]. Salmonella is the most common causative agent $[1,2]$. The clinical presentation is typically fever with a painful pulsatile abdominal mass. General management involves antibiotics, surgical debridement with or without revascularization, or endovascular treatment [3]. Multidetector computed tomography angiography (CTA) is the first modality for the diagnosis of an infected aneurysm. Radionuclide scanning is generally reserved for the detection of vascular graft infection; however, it can also be used in the diagnosis of infected aneurysm [4].

Gallium-67 scans have long been proven useful in localizing infection in infected aneurysms or vascular graft infections [5,6], particularly for evaluating the diagnosis, extent of disease, and efficiency of treatment [7]. The combined use of gallium-67 single photon emission computed tomography (SPECT) with contrast computed tomography (CT) has been shown to be useful in evaluation since 1990, with added specificity [8]. 
The use of functional imaging in the evaluation of infected aneurysms is currently a trend for the evaluation of the disease, as conventional imaging cannot gauge metabolic activity [9], which can be addressed by functional imaging [10]. Various recent case reports have demonstrated efficacy in its diagnosis $[9,11]$. However, there is a lack of knowledge regarding the further evaluation and monitoring of this group of patients. Previous case reports have mainly focused on the initial diagnosis. Relapse of infection is common and was reported in up to $42.6 \%$ of cases [1]. The optimal imaging techniques for postoperative evaluation, monitoring of endovascular grafts, and how imaging may guide further management remain uncertain. Therefore, we would like to report our case series over 10 years regarding the use of gallium-67 SPECT with contrast CT as a functional imaging adjunct to conventional CTA.

\section{MATERIALS AND METHODS}

We performed a retrospective cohort analysis of prospectively collected data involving all patients with an infected abdominal aortic aneurysm (IAAA) referred to a radiology unit from Princess Margaret Hospital in Hong Kong between January 2010 and December 2020. The patients were identified using the Radiology Information System. The Institutional Review Board of the Radiology Department approved the study design and use of clinical data (approval number: KW/EX-19-061[137-02]). The written informed consent was waived by the board.

For gallium-67 SPECT with contrast CT, the CT model was Discovery NM/CT 670 (GE Healthcare, Chicago, IL, USA) with the administration of gallium-67 Citrate of 3 mci (adult dose). Images were obtained at 48-h post-injection using a dual-head SPECT-CT camera with medium energy general purpose collimators. We then performed a whole body scan at $10 \mathrm{~cm} / \mathrm{min}$, and then SPECT on the abdomen by a total of 60 pairs of views, each of $25 \mathrm{~s}$ duration, acquired over $360^{\circ}$ in a $128 \times 128$ matrix. CT was performed with noncontrast, arterial, and venous phase CT images (helical, 120 $\mathrm{kV}$; slice, $1.25 \mathrm{~mm}$; pitch, 1; rotation, $0.5 \mathrm{~s}$; intravenous (IV) contrast, $70 \mathrm{~mL}$ at a rate of $3 \mathrm{~mL} / \mathrm{s}$ ). SPECT CT reconstruction was performed using ordered 3-dimensional (3D) subset expectation maximization with two iterations, 16 subsets, and a Butterworth filter by GE Xeleris Functional Imaging Workstation (GE Healthcare).

For CT angiogram pre- and post-endovascular aortic repair (EVAR) scans, the CT model was Somatom Definition Flash VA48A (Siemens Healthcare GmbH, Erlangen, Germany). Scan parameters included helical Combined Applications to Reduce Exposure kV; slice, $0.6 \mathrm{~mm}$; pitch, 0.8 ; rotation, $0.5 \mathrm{~s}$. IV contrast $70 \mathrm{~mL}+30 \mathrm{~mL}$ saline was ad- ministered at a rate of $3 \mathrm{~mL} / \mathrm{s}$. For pre-EVAR cases, we performed the arterial phase only, while for post-EVAR cases, both arterial and venous phases were performed. 3D reconstruction was performed using Syngo CT VA48A (Siemens Healthcare $\mathrm{GmbH}$ ).

Cases with proven IAAA were followed up annually by CTA with gallium-67 SPECT as per the local hospital's protocol. All planar, SPECT, and CTA images were reviewed and interpreted by two radiologists with more than 10 years of nuclear medicine experience. Diagnosis was made based on the consensus of both. Positive findings suggesting infection on SPECT were defined as follows: focal increased uptake in the graft or aneurysmal tissue, site of previously infected AAA, periaortic soft tissue, or psoas muscle. The focal uptake is correlated to the respective CTA image for the corresponding soft tissue changes.

\section{RESULTS}

A total of five patients were identified (age at presentation, 63 to 74 years; four males and one female). The diagnosis was confirmed either by imaging or surgical specimens, as supported by microbiological studies (Table 1). Four patients had a primary infected abdominal aneurysm, whereas the fifth had a secondary infected vascular graft for AAA (Fig. 1-4).

Relapsed infection was defined as the occurrence of a continuous fever ( $>3$ days), sepsis, graft or stent-graft infection, abscess, multiple organ dysfunction syndrome, aorto-enteric fistula, gastrointestinal bleeding, or recurrent IAAA [12]. It appeared in two out of the five cases (Fig. 1, 3), ranging from 9 months to 4 years after the initial episode. This was confirmed by both CTA and gallium-67 SPECT with contrast $\mathrm{CT}$, in which functional imaging demonstrated its ability for early detection of relapsed infection with accurate extent characterization. The extent of infection also involved adjacent contiguous structures (such as spinal infection with paravertebral soft tissue and psoas abscess) in two cases, which were confirmed by functional imaging. All relapsed infections required urgent aggressive treatment with broad-spectrum IV antibiotics. Image-guided drainage of psoas abscesses was offered in a timely manner, in addition to broad-spectrum IV antibiotic therapy for controlling sepsis. Resolved infections were confirmed by functional imaging in these cases, which allowed switching from a parental to oral antibiotic regimen and discharge of the patients.

Four patients currently have shown no evidence of active infection on routine surveillance imaging with CTA and interval gallium-67 SPECT with contrast CT. They survived for 7 to 10 years from the first presentation and were 
Table 1. Patient demographics

\begin{tabular}{|c|c|c|c|c|c|c|}
\hline Case & Age (y) & Sex & Microbiology & Use of Ga/SPECT & Outcome/antibiotics & Endoleak on CTA \\
\hline 1 & 65 & M & Salmonella (blood culture) & $\begin{array}{l}\text { Detection of relapsed infection } \\
\text { Confirming infection resolution } \\
\text { Monitoring }\end{array}$ & $\begin{array}{l}\text { Resolved infection/lifelong } \\
\text { prophylaxis }\end{array}$ & No \\
\hline 2 & 66 & $\mathrm{~F}$ & Negative & $\begin{array}{l}\text { Confirming infection resolution } \\
\text { Monitoring }\end{array}$ & $\begin{array}{l}\text { Resolved infection/lifelong } \\
\text { prophylaxis }\end{array}$ & No \\
\hline 3 & 63 & M & Salmonella (blood culture) & $\begin{array}{l}\text { Early detection of relapsed periaortic } \\
\text { graft infection } \\
\text { Detection of paraspinal abscess with } \\
\text { extent localization }\end{array}$ & $\begin{array}{l}\text { Lifelong antibiotic therapy } \\
\text { instead of surgery }\end{array}$ & No \\
\hline 4 & 69 & M & Negative & $\begin{array}{l}\text { Detection of infected graft } \\
\text { Confirming resolution }\end{array}$ & $\begin{array}{l}\text { Resolved infection/lifelong } \\
\text { prophylaxis }\end{array}$ & No \\
\hline 5 & 74 & M & Klebsiella (aneurysmal tissue) & $\begin{array}{l}\text { Evaluation for infection foci at aorta/ } \\
\text { around aorta }\end{array}$ & $\begin{array}{l}\text { Succumbed } 2 \text { months } \\
\text { post-operatively }\end{array}$ & No \\
\hline
\end{tabular}

Ga/SPECT, gallium-67 single photon emission computed tomography; CTA, computed tomography angiography; $M$, male; $F$, female.
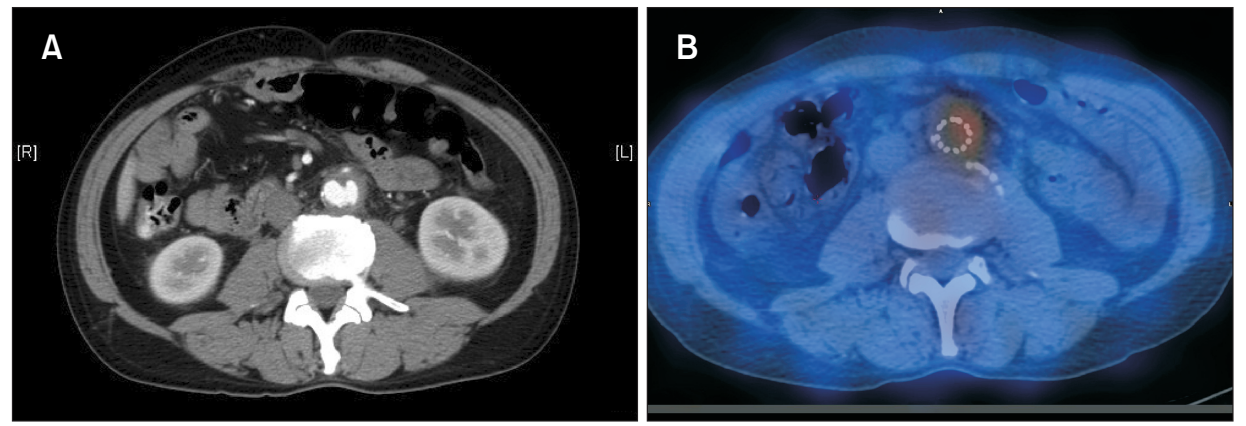

Fig. 1. Images of Case 1. (A) Computed tomography angiography (CTA) showed an infected abdominal aortic aneurysm with impending rupture. (B) Gallium-67 single photon emission computed tomography (SPECT) with contrast computed
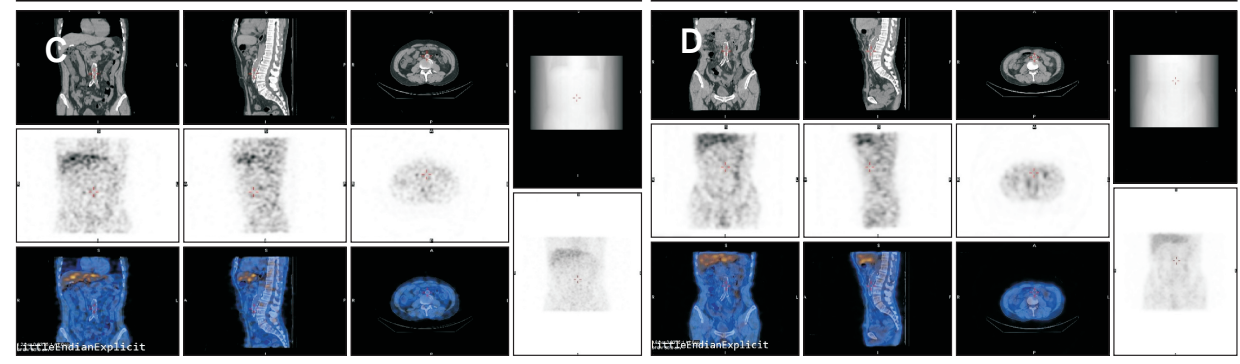
tomography performed 4 years later showed increased focal tracer uptake. (C) Follow-up CTA and gallium-67 SPECT after treatment showed resolution of infection. (D) Interval surveillance using gallium-67 SPECT confirmed no relapse of infection.

administered lifelong prophylactic antibiotics. Patient 5 succumbed shortly from the underlying IAAA with postoperative complications. No patient had an endoleak on CTA.

\section{1) Case 1}

A 65-year-old male with good health presented with fever and abdominal pain for a few days. White blood cell count was normal upon admission $(9,200 / \mu \mathrm{L})$. The patient was diagnosed with an IAAA with impending rupture on CTA. He was treated with EVAR (Endurant; Medtronic, Minneapolis, MN, USA) and femoral-femoral crossover bypass in 2010. Blood culture yielded Salmonella group D. He was administered a 2-month course of IV ceftriaxone $1 \mathrm{~g}$ every 12 hours and oral ciprofloxacin $250 \mathrm{mg}$ twice daily, followed by oral azithromycin $500 \mathrm{mg}$ daily. Serial followup gallium-67 SPECT showed resolution of the inflammatory focus until 6-month postoperatively. Antibiotics were stopped because of the resolution of inflammatory changes by gallium-67 SPECT. Gallium-67 SPECT with contrast CT 4 years later showed increased focal tracer uptake (Fig. 1B), confirming relapsed infection with suggestive findings of periaortic fat stranding on CT. He had mild abdominal pain and mild leukocytosis $(14,300 / \mu \mathrm{L})$. Blood culture results were negative. He subsequently received a 3-month course of $\mathrm{IV}$ antibiotics (ceftriaxone $2 \mathrm{~g}$ daily with oral azithromycin $500 \mathrm{mg}$ daily), with a follow-up CTA and gallium-67 SPECT after treatment showing resolved infection (Fig. 1C). 

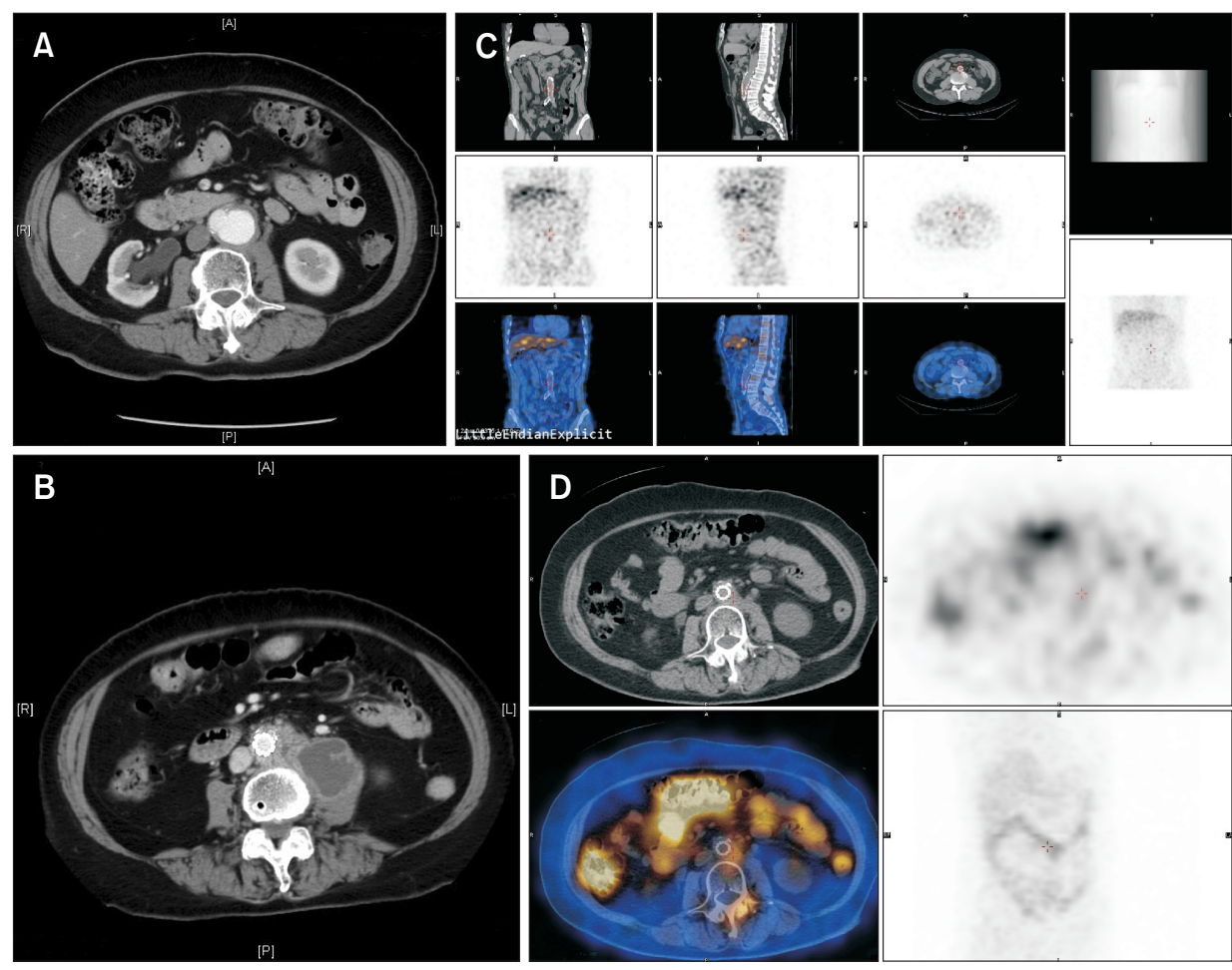

D
Fig. 2. Images of Case 2. (A) Computed tomography (CT) showed a rapidly enlarging infected abdominal aortic aneurysm. (B) Follow-up CT showed relapse of periaortic infection with a left psoas abscess. (C) Follow-up gallium-67 single photon emission computed tomography (SPECT) with contrast CT 4 months later showed residual inflammatory/infective changes. (D) Follow-up gallium-67 SPECT with contrast CT in 2016 showed resolution of infection.
A joint decision to administer life-long prophylactic antibiotic (azithromycin $500 \mathrm{mg}$ daily) was made by vascular surgeons and microbiologists. Serial follow-up CTs from 2015 to 2019 showed no endoleaks or relapse of infection, and interval surveillance gallium-67 SPECT with contrast CT in 2017 confirmed no relapse of infection (Fig. 1D). The patient opted to continue conservative treatment, and he has had no detectable recurrence since then.

\section{2) Case 2}

A 66-year-old female was incidentally diagnosed with rapidly enlarging IAAA on CT for follow-up of renal stones in 2012 (Fig. 2A), and was treated with EVAR (Zenith graft; Cook Medical LLC, Bloomington, IN, USA). Leukocytosis $(15,900 / \mu \mathrm{L})$ was observed. Blood culture results were negative. A CT performed in 2014 showed a relapse of periaortic infection with a left psoas abscess (Fig. 2B). Leukocytosis $(11,500 / \mu \mathrm{L})$ was seen, as well as an elevated C-reactive protein level of $123 \mathrm{mg} / \mathrm{L}$. The patient was treated with CTguided drainage of the left psoas abscess and IV antibiotic (initial 3-day course of piperacillin-tazobactam $4.5 \mathrm{~g} \mathrm{ev-}$ ery 8 hours followed by an 8 -week course of amoxicillinclavulanate [1.2 g] every 8 hours). Blood culture results were negative. Follow-up gallium-67 SPECT with contrast CT 4 months later showed residual inflammatory/infective changes (Fig. 2C). A follow-up gallium-67 SPECT with contrast CT in 2016 showed resolution of infection (Fig. 2D).
There was no relapsed infection detected on surveillance CT. She was administered a lifelong antibiotic (oral levofloxacin $250 \mathrm{mg}$ daily).

\section{3) Case 3}

A 63-year-old male presented with fever and abdominal pain, with CT pointing to possible IAAA in 2013 (Fig. 3A), which yielded Salmonella group D. He was treated with an aortic stent graft (Endurant 11; Medtronic) (Fig. 3B) and IV antibiotics (2-month course of ceftriaxone $2 \mathrm{~g}$ daily followed by a 4-month course of azithromycin $500 \mathrm{mg}$ daily). A follow-up gallium-67 SPECT with contrast CT 3 months after interval shrinkage of the aneurysm showed residual inflammatory changes at the aortic aneurysmal sac and at the area adjacent to the stent graft. Further follow-up gallium-67 SPECT with contrast CT in early 2014 showed resolution of inflammation. However, the first breakthrough infection occurred 9 months later, and urgent CT for fever and back pain showed infective collection around the distal end of the aortic stent-graft. Blood culture results were negative. After treatment, the follow-up CT after 1 month showed interval resolution of the infective collection. The second breakthrough infection occurred in 2015. The follow-up gallium-67 SPECT in January 2015 with contrast CT identified new inflammation/infection foci adjacent to the stent graft (Fig. 3C). At that time, the patient was reviewed, and was found asymptomatic and afebrile, with his white 



Fig. 3. Images of Case 3. (A) Computed tomography (CT) showed an infected aneurysm.
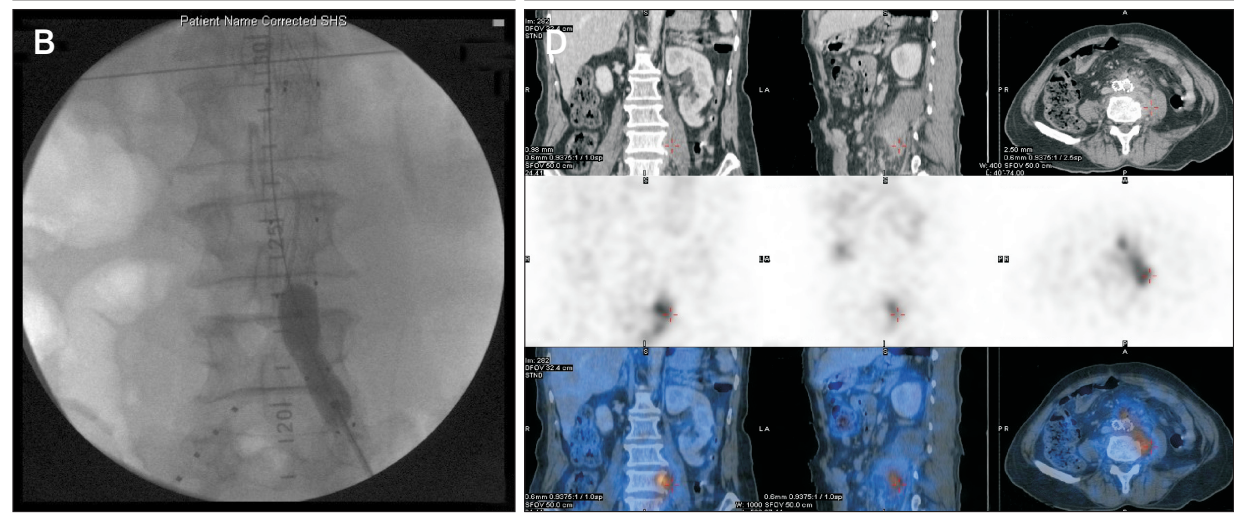
(B) An aortic stent graft was deployed. (C) Follow-up gallium-67 single photon emission computed tomography (SPECT) identified new inflammation/ infection foci adjacent to the stent graft. (D) Another gallium-67 SPECT with contrast CT showed an infective lesion anterior to the aortic bifurcation and left common iliac artery with a paraspinal abscess.

cell count being normal $(8,000 / \mu \mathrm{L})$ and CRP level mildly elevated $(8.2 \mathrm{mg} / \mathrm{L})$. CTA confirmed the infective collection at the distal end of the stent graft. He was administered IV ceftriaxone $4 \mathrm{~g}$ daily and oral levofloxacin $250 \mathrm{mg}$ twice per day. Initially, there was no fever, but he eventually became febrile and developed sepsis as well as leukocytosis $(16,500 / \mu \mathrm{L})$ in late June 2015 . Another follow-up gallium-67 SPECT with contrast CT done in July 2015, which was 6 months after the last study, showed an infective/inflammatory lesion anterior to the aortic bifurcation and left common iliac artery, causing tethering of mesenteric vessels and obstruction of the left ureter, with paraspinal extension causing a small paraspinal abscess at $\mathrm{L} 4$ and erosion of the L4 and L5 cortices (Fig. 3D). Oral metronidazole (400 mg, three times daily) was added in addition to the previous regimen. White cell counts and inflammatory markers were normalized. Blood cultures results were negative. CT-guided drainage of the collection was not performed because of technical difficulty. Two months after treatment, the follow-up CT showed similar soft tissue stranding and inflammatory/infective changes around the aortic bifurcation, but with resolution of the rim-enhancing fluid collection. The same antibiotic regimen was continued for 2 months. The third breakthrough infection occurred with recurrence of the rim-enhancing collection adjacent to the $\mathrm{L} 4$ vertebral body 3 months later upon repeat CT in 2015. IV ertapenem (1 $\mathrm{g}$ daily) and oral co-trimoxazole (960 mg, twice daily) were administered. White cell counts and inflammatory markers were normalized. This antibiotic regimen was continued for 8 months before stepping down to oral azithromycin (500 mg daily). Subsequent follow-up CTs from 2016 to 2018 showed persistent periaortic inflammatory changes with an enlarging aneurysm. The patient opted for long-term oral antibiotic prophylaxis therapy (combined azithromycin 500 $\mathrm{mg}$ daily plus co-trimoxazole $960 \mathrm{mg}$ twice per day) with interval monitoring instead of surgical exploration. The latest gallium-67 SPECT with contrast CT in 2020 showed no disease recurrence.

\section{4) Case 4}

A 69-year-old male with good past health presented with a 1-week history of abdominal pain and low grade fever. Mild leukocytosis $(11,300 / \mu \mathrm{L})$ was observed on admission. A 5.3-cm infrarenal abdominal aortic aneurysm with impending rupture was seen on CTA in 2013 (Fig. 4A), with endovascular repair (Endurant; Medtronic) (Fig. 4B). Blood culture results were negative. Gallium-67 SPECT with contrast CT showed focal uptake, thus confirming the diagnosis (Fig. 4C). He was treated with oral antibiotics (a 3-week course of amoxicillin-clavulanate $375 \mathrm{mg}$ three times daily followed by a 17-week course of levofloxacin $750 \mathrm{mg}$ daily), and the follow-up gallium-67 SPECT with contrast CT 3 months later showed largely resolved uptake, suggesting re- 

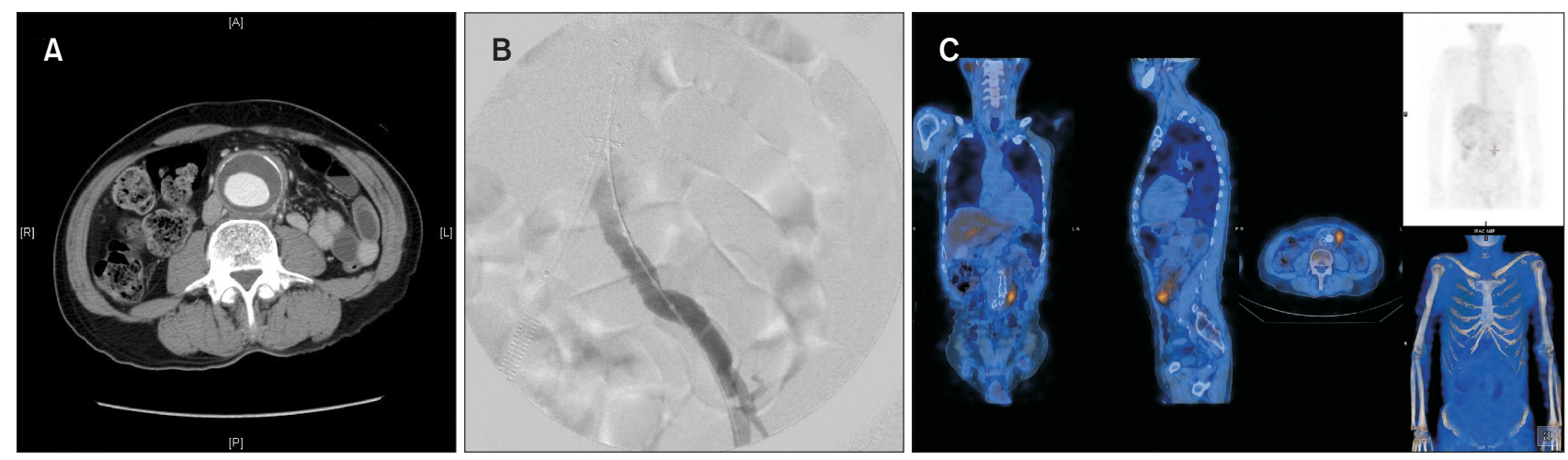

Fig. 4. Images of Case 4. (A) Computed tomography (CT) angiography showed a 5.3-cm infrarenal abdominal aortic aneurysm with impending rupture. (B) Endovascular repair was performed. (C) Gallium-67 single photon emission computed tomography with contrast CT showed focal uptake, thus confirming the diagnosis of an infected aneurysm.

solved infections. A follow-up CT in 2016 also revealed no changes in size or any no infective/inflammatory changes of the aortic aneurysm. He was prescribed lifelong oral prophylactic antibiotic (cefuroxime $250 \mathrm{mg}$ daily).

\section{5) Case 5}

A 74-year-old male with secondary infrarenal AAA with known $\mathrm{L} 4 / 5$ spondylodiscitis presented with acute abdominal pain with a ruptured AAA confirmed on ultrasonography. On the same day, emergency surgery was performed with resection of the ruptured AAA and graft replacement, which required massive transfusion with inotropic support during the operation. Culture of the aneurysmal wall tissue showed growth of Klebsiella. He was administered IV antibiotics (vancomycin $250 \mathrm{mg}$ every 8 hours and sulperazon $1 \mathrm{~g}$ every 12 hours). Follow-up CT on postoperative day 12 showed AAA post-graft placement and left retroperitoneal hematoma, as well as a large complex multiloculated retroperitoneal collection and persistent $\mathrm{L} 4 / 5$ spondylodiscitis. He underwent a second laparotomy and drainage. Postoperatively, he developed pneumonia, Candida fungemia probably secondary to line sepsis, and abdominal compartment syndrome. Gallium-67 SPECT with contrast CT performed 2 months after the initial operation showed interval shrinkage of the aneurysmal sac and mild gallium uptake in the anterior portion of the AAA, suggesting an underlying infection/inflammatory change. He went into cardiac arrest, but was resuscitated and given immediate care. Another repeated blood culture also showed Klebsiella growth, with the patient succumbing 1 month later from deconditioning.

\section{DISCUSSION}

IAAA is a life-threatening condition requiring urgent intervention, such as endovascular repair or open surgery. A significant reinfection rate of up to $42.6 \%$ has been reported [3]. In general, there was a lack of knowledge in the follow-up evaluation of these patients, especially when they had an endograft in situ. A further step is to consider the management of these patients, particularly determining whether lifelong prophylactic antibiotic is needed. The strongest predictor of poor long-term outcomes is persistent infection, with a 1-year survival rate of only 39\% [13]. Therefore, metabolic imaging, such as gallium-67 SPECT with contrast CT plays a crucial role in the follow-up evaluation of IAAA, which would allow detection of persistent infection or reinfection and ensure complete remission of infective activity.

Gallium-67 SPECT with contrast CT, together with CTA, have promising roles in confirming a diagnosis of IAAA. This is in concordance with previous studies $[3,13]$. This combination technique demonstrated its usefulness in the detection of relapsed infection with accurate localization and follow-up to check for complete resolution of infection activity. This allows early detection of re-infection with accurate characterization. As demonstrated in case 3, timely treatment could be offered to patients with the confirmation of abnormal metabolic activity indicating infection. In addition, concomitant use of CTA for follow-up evaluation is very helpful in detecting endoleaks from the aortic stent graft.

Gallium-67 SPECT with contrast CT provides invaluable information for guiding management during follow-up evaluations in the long run. First, it allows triage of patients by correlating metabolic activity with treatment urgency and strategy. A metabolically active disease suggests active infection/inflammation, which requires urgent and timely stepping up of broad-spectrum antibiotic therapy. Metabolically active fluid collection requires image-guided or 
surgical drainage. IAAA requires multidisciplinary management, especially when contagious spread of infection takes place (e.g., to the spine causing spondylodiscitis). Additional input by orthopedic surgeons and microbiologists in patient management is necessary. Resolution of metabolic activity confirms resolved infective/inflammatory changes, and adjustment of antibiotics can be performed accordingly. Therefore, it provides a guide for successful treatment, deciding the duration of treatment, or whether these patients need lifelong prophylactic antibiotic. Gallium scans help localize the site of inflammation. They are less expensive and do not require special preparation [7]. Concerns about the unreliability of using gallium scans alone for imaging abdominal infection due to physiological bowel activity have been discussed [5]. However, the potential drawbacks of non-specific uptake at the bowels, liver, and spleen could be overcome by using SPECT, which offers accurate localization and confirmatory diagnosis along with CTA.

Luo et al. [3] reported the successful use of gallium-67 SPECT for the accurate localization of IAAA, thus allowing timely and accurate diagnosis. In the cases in the present study, the use of hybrid imaging not only allowed for timely diagnosis, but also the monitoring and early detection of reinfection, thereby guiding treatment. The latest European Society for Vascular Surgery guidelines on the management of IAAA recommend 6 to 12 months or lifelong postoperative antibiotic treatment [14], which is formulated on a case-by-case basis in close collaboration with microbiology specialists based on clinical and laboratory parameters and imaging studies [14]. The current study's illustration also sheds light on this management decision in deciding the duration of antibiotic therapy. We suggest incorporating gallium-67 SPECT with CTA in the routine follow-up protocol (e.g., yearly).

Compared to other metabolic imaging modalities, the Indium-111 white blood cell scan is more expensive than the Gallium-67 scan [8], but it could be performed 18 to 24 hours after injection, presenting an advantage over the latter. However, this was not available in our hospital. ${ }^{18} \mathrm{~F}-$ fluorodeoxyglucose positron emission tomography/CT (18 FDG-PET/CT) is a potential alternative for confirming the diagnosis and monitoring response to antibiotics $[9,15]$. The main advantages of gallium-67 scan over 18 FDG-PET/CT are its lower cost and local availability. In Hong Kong, only a minority of hospitals are equipped with 18 FDG-PET/CT machines, and are dedicated to oncology services most of the time. Gallium-67 SPECT with contrast-enhanced CT is more readily available. One potential drawback of gallium-67 SPECT with contrast CT is the higher radiation dose received by patients, but it is relatively inexpensive compared to 18 FDG-PET/CT.
The retrospective observational nature of this study was a major limitation. Other limitations include the study's small sample size due to the rarity of the disease. In addition, there is no optimal cutoff value nor standardized protocol for gallium uptake. Furthermore, gallium-67 SPECT is not readily available in urgent settings because of the lack of related expertise and cost of study. A higher radiation dose is also associated with the use of gallium-67 SPECT compared with gallium studies, but the potential advantage of accurate disease localization and treatment implications renders the procedure worthwhile.

Multicenter, large scale, prospective studies comparing hybrid imaging and conventional imaging can further prove their usefulness. Furthermore, comparisons of different metabolic imaging modalities may also be helpful. It is hoped that optimal follow-up protocols and management protocols will be elucidated further in the near future.

\section{CONCLUSION}

The use of gallium-67 SPECT with contrast CT is useful in the evaluation and monitoring of patients with IAAA, and serves an important role in guiding long-term management.

\section{CONFLICTS OF INTEREST}

The authors have nothing to disclose.

\section{ORCID}

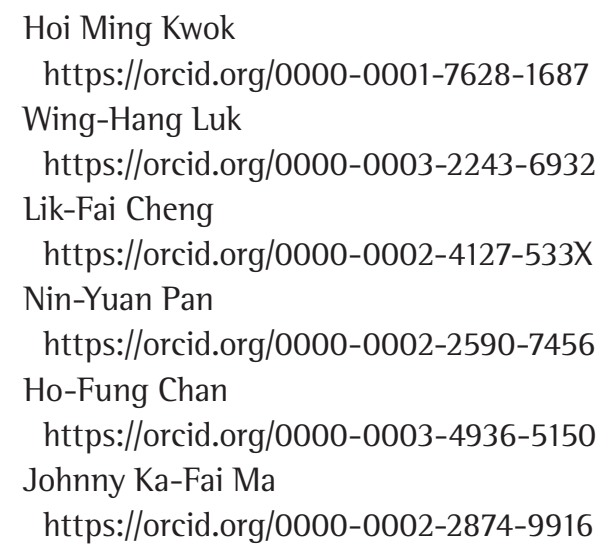

\section{AUTHOR CONTRIBUTIONS}

Concept and design: HMK, WHL. Analysis and interpretation: HMK, WHL. Data collection: HMK, WHL. Writing the article: HMK. Critical revision of the article: all authors. Final approval of the article: all authors. Statistical analysis: none. Obtained funding: none. Overall responsibility: HMK. 
1) Murphy DJ, Keraliya AR, Agrawal MD, Aghayev A, Steigner ML. Crosssectional imaging of aortic infections. Insights lmaging 2016;7:801-818.

2) Lee WK, Mossop PJ, Little AF, Fitt GJ, Vrazas Jl, Hoang JK, et al. Infected (mycotic) aneurysms: spectrum of imaging appearances and management. Radiographics 2008;28:1853-1868.

3) Luo Y, Zhu J, Dai X, Fan H, Feng Z, Zhang $\mathrm{Y}$, et al. Endovascular treatment of primary mycotic aortic aneurysms: a 7-year single-center experience. J Int Med Res 2018;46:3903-3909.

4) Deipolyi AR, Rho J, Khademhosseini A, Oklu R. Diagnosis and management of mycotic aneurysms. Clin Imaging 2016;40:256-262.

5) Orton DF, LeVeen RF, Saigh JA, Culp WC, Fidler JL, Lynch TJ, et al. Aortic prosthetic graft infections: radiologic manifestations and implications for management. Radiographics 2000;20:977-993.

6) Hsu CC, Huang YF, Chuang YW. Detection of an infected abdominal aortic aneurysm with three-phase bone scan and gallium-67 scan. Clin Nucl Med 2008;33:305-307.

7) Banzo I, Quirce R, Serrano J, Jimenez J, Tabuenca 0, Carril JM. Ga-67 citrate scan in vascular graft infection. Ann Nucl Med 1992;6:235-239.

8) Johnson KK, Russ PD, Bair JH, Friefeld GD. Diagnosis of synthetic vascular graft infection: comparison of CT and gallium scans. AJR Am J Roentgenol 1990;154:405-409.

9) Bedmutha AS, Singh N, Shivdasani D. Metabolic imaging as a novel strategy in evaluation of mycotic abdominal aortic aneurysm: a case report and brief clinical review. Indian J Nucl Med 2017;32:336-339.

10) Ishizaka N, Sohmiya K, Miyamura M, Umeda T, Tsuji M, Katsumata T, et al. Infected aortic aneurysm and inflammatory aortic aneurysm--in search of an optimal differential diagnosis. J Cardiol 2012;59:123-131.

11) Cheng MF, Liu KL, Lin YF, Tzen KY, Yen RF. 67Ga SPECT/CT aids in the diagnosis of occult infected common iliac artery aneurysm. Clin Nucl Med
2013;38:573-575

12) Liu M, Liu P, Liu B, Che H, Liu J, Sun A, et al. Infection-relapse and a potentially surgical stratification model for the treatment of mycotic aortic aneurysms: a propensity-matched pilot study. Vascular 2019;27:500-510.

13) Kan CD, Lee HL, Yang YJ. Outcome after endovascular stent graft treatment for mycotic aortic aneurysm: a systematic review. J Vasc Surg 2007;46:906-912.

14) Wanhainen $A$, Verzini $F$, Van Herzeele 1, Allaire E, Bown M, Cohnert T, et al. Editor's choice - European Society for Vascular Surgery (ESVS) 2019 clinical practice guidelines on the management of abdominal aorto-iliac artery aneurysms. Eur J Vasc Endovasc Surg 2019;57:8-93.

15) Choi SJ, Lee JS, Cheong MH, Byun SS, Hyun IY. F-18 FDG PET/CT in the management of infected abdominal aortic aneurysm due to Salmonella. Clin Nucl Med 2008;33:492-495. 